\title{
Refined garbage collection for open distributed systems with multicapabilities
}

\begin{abstract}
Capabilities can provide information not only on a particular object, but also on which methods of the object an agent is permitted to invoke. Specific information about an agentó óknowledgeô is potentially very useful and can be manipulated in a variety of ways. This paper focuses on the LINDA coordination model of open distributed systems. One limited resource is memory, and garbage collection has already been proposed for the standard LINDA with multiple tuple-spaces (TSs) to avoid memory exhaustion. The implementation, however, was restricted to garbage collection of TSs. Taking into account the need for garbage collection not only for TSs, but also for tuples, this paper demonstrates how the garbage collection mechanism can be extended to handle unusable tuples, with the introduction of multicapabilities, which generalise capabilities to collections of objects.
\end{abstract}

Keyword: Distributed systems; LINDA; Garbage collection; Tuple-spaces (TSs) 